\title{
Attentional requirements of walking in spinal cord injured patients compared to normal subjects
}

\author{
Y Lajoie*,1, H Barbeau ${ }^{2}$ and M Hamelin ${ }^{3}$ \\ ${ }^{1}$ School of Human Kinetics, Laurentian University, Sudbury, Canada; ${ }^{2}$ School of Physical and Occupational Therapy, \\ McGill University, Montréal, Canada; ${ }^{3}$ Laboratoire de Performance Motrice Humaine, Laval University, Quebec, \\ Canada
}

\begin{abstract}
Study Design: Recent developments in the field of rehabilitation and the increase in the number of incomplete spinal cord injured subjects (SCI) emphasize the importance of studying the walking behavior of that population.

Objectives: Attentional requirements were measured in spinal cord injured subjects during sitting, standing and walking and compared to a healthy group for the same tasks and walking at a similar speed.

Methods: Auditory stimuli and verbal responses were used to measure the attentional demands in the three experimental tasks.

Results: For both static tasks, SCI subjects were faster than the normal group. During walking at preferred speed, the normal group is significantly faster than the SCI subjects are. No difference was found between the two groups when the normal group walked at a similar speed. However, SCI subjects need to allocate significantly more attentional resource to walking than the normal. SCI patients also showed slower reaction times when the stimuli were presented during the single-support phase of walking. Kinematics analysis revealed that SCI subjects produced longer cycle duration than the normal group even when they walked at a match speed. Although variability in the cycle duration and the cycle length were comparable between the two groups, when the normal group walked at a slower speed, they were significantly more variable than the SCI subjects.

Conclusion: Walking for SCI patients is cognitively challenging. Walking speed seems to be an important factor associated with attentional demands.
\end{abstract}

Keywords: attentional demands; walking; spinal cord injured patients; reaction times

\section{Introduction}

Recent developments in the field of rehabilitation using medication, locomotor training with weight support, locomotor training with functional electrical stimulation and their combination (see Barbeau et $a l^{1,2}$ for a review) have led to increasing locomotor capabilities of patients who have sustained a spinal cord injury (SCI). According to Burke et $a l^{3} 39 \%$ of a sample of 262 discharged SCI patients were functional walkers, $37 \%$ could not walk and $24 \%$ could walk but normally use a wheelchair for daily activities. Thus emphasizing the importance of studying walking, to help the development of new approaches to rehabilitation.

Walking is a highly practiced activity, and has often been considered as an automatic behavior. Animal studies (cat) isolating the spinal cord from the brain, revealed that it is possible to observe some walking

*Correspondence: Y Lajoie, Laurentian University, School of Human Kinetics, Ramsey Lake Road, Sudbury, Ontario, Canada, P3E 2C6, Canada abilities without the presence of supraspinal mechanisms (see Rossignol et $a l^{4}$ for a review). Nevertheless, walking should not be considered only as an automatic task, but rather as a build up of different control levels. Forssberg ${ }^{5}$ and Grillner et $a l^{6}$ have identified three prerequisites that rehabilitation should work on to help the recovery of locomotor abilities. These are: (1) basic or stereotyped movement synergy to achieve propulsion, (2) maintenance of equilibrium during propulsive movement and (3), adaptation of locomotor pattern to the behavioral goals of the person and the constraints of the environment. In addition, supraspinal mechanisms could be emphasized, such as mechanisms responsible for the integration of several types of sensory information (visual, vestibular and proprioceptive), and cognitive involvement required to fine tune the movements and the selected appropriate behaviors.

The involvement of the cognitive level in human locomotion has recently been studied in normal and pathological populations. Lajoie et $a l^{7}$ have shown that 
normal walking requires more attentional requirements in normal subjects than sitting and standing. Lajoie et $a l^{8}$ in a case study of a deafferented patient have shown that walking could be achieved without afferent information from the limbs. In this patient, only the sensory level was affected while the motor level was intact. The deafferented patient needs to use substantially more attention than normal to walk because he must rely on an exocentric frame of reference and external sources of feedback to determine his position in space during the movement. Furthermore, they showed that proprioception of the neck may play an important role in reestablishing a referential guidance platform around which the mobile limbs might securely move. They noted that another patient deprived of neck proprioception was not able to walk without a stable assistive device such as parallel bars or walker. Those studies emphasized that walking is not purely automatic from a cognitive standpoint. Furthermore, locomotion could still be achieved even when proprioception is weakened but with the need of more cognitive involvement.

In pathological walking in a spinal cord injured patient (SCI), spasticity, clonus, weakness and postural instability may result in a more complex walking pattern and might require far more information processing than a normal subject (see Barbeau et $a l^{9}$ for a review). Those constraints prevent fluid and natural walking and patients must generate adaptations that could affect the cognitive demands of the walking task. Since the attentional capacity is limited, this increase in cognitive demands might be sufficient to decrease the patient's security where he could not be able to correctly integrate stimuli from his environment.

In this study we evaluate the attentional requirement of SCI patients during sitting and during walking in order to measure the attentional involvement related to walking in comparison to normal subjects. Furthermore, because it has been shown that speed plays a major role in kinematic variables and equilibrium constraints, attentional demands when walking at a patient's match speed will also be evaluated.

\section{Methods}

Six subjects suffering from incomplete spinal cord injury and ten healthy young adults (mean age $=25$ ) were studied (Table 1). All were clinically stable at the time of testing, and all showed signs of hyperactive stretch responses in the triceps surae muscle group. Some were on medications such as baclofen and cyproheptadine to reduce these responses. All patients and normal subjects gave informed consent. The ambulatory ability for the patients varied; two of them walked with canes, three with Canadian crutches and one without assistive device.

The subjects walked on a 6-m length of pathway providing the recording of at least five walking cycles. The subject's shoes were instrumented with pressure switches (Interlink Electronics, California) placed under the heel and toes of each foot. These contacts were digitally coded to provide accurate temporal values corresponding to the onset and offset of right and left single-support and double-support phases. The horizontal foot displacement of the two feet was recorded via small monofilament wires attached to the rear of each shoe. Each wire was wrapped around a plastic wheel $(30-\mathrm{cm}$ circumference) fixed onto a gearbox system having a 4.6:1 ratio. A ten turn high precision potentiometer was mounted on the shaft of the rotating axis and provided a voltage proportional to the distance covered. The wires were maintained stable with a constant resistance spring $(0.66 \mathrm{~N})$ fixed into the rotating mechanisms. This low resistance served only to prevent the wires from shivering; it did not affect the walking and was not perceived by the subject. The system provided a resolution of $3 \mathrm{~mm}$.

To evaluate the mental load required for locomotion, the subjects walked at their preferred pace and were asked to consider the walking task the primary task. The secondary task was to respond as rapidly as possible to an auditory stimulus $(1 \mathrm{KHz}, 50 \mathrm{~ms}$ duration) with a vocal response ('top'). The subjects wore a helmet equipped with a microphone. The analog signal from the microphone was used to detect the onset of the verbal response. Reaction times (RTs) were evaluated by computing the temporal difference between the presentation of the stimuli and the onset on the verbal responses. Without going into the specific details of cognitive resource models, when the primary task (walking in the present experience) is unaffected by the introduction of a probe reaction time task (discrete secondary task, consisting of a simple reaction time to a stimulus), an increased probe

Table 1 Demographic information on the patients

\begin{tabular}{lcccccc}
\hline Code & Age & Sex & Diagnosis & $\begin{array}{c}\text { Time since } \\
\text { injury (years) }\end{array}$ & Foot contact & Ambulatory status \\
\hline 1 & 36 & M & T T12-L1 & 15 & HC & Independent \\
2 & 30 & M & T C5-C6 & 10 & TC & 2 Canadian crutches \\
3 & 51 & F & T C5-C6 & 6 & LHC, RTC & 2 Canes \\
4 & 30 & M & T C6 & 13 & TC & 2 Canadian crutches \\
5 & 38 & M & T C6 & $>20$ & TC & 2 Canadian crutches \\
6 & 43 & M & T T2-T10 & 6 & HC & Cane \\
\hline
\end{tabular}

$\mathrm{TC}=$ toe contact; $\mathrm{HC}=$ heel contact; $\mathrm{LHC}=$ left heel contact; $\mathrm{RTC}=$ right toe contact; $\mathrm{T}=$ thoracic 
reaction time reflects an increased attentional load. ${ }^{10,11}$ Signals from foot contacts and the microphone were sampled at $500 \mathrm{~Hz}$. Before starting the walking condition, the subjects were familiarized with the walking environment. After these trials, data were collected for five trials without the secondary task (control condition). These trials served to establish the walking pattern of the subjects. For the next 20 trials, stimuli were randomly presented at the onset of the single support condition (SS) of the second (four trials) or third (four trials) walking cycle or at the onset of the double support condition (DS) of the second (four trials) or third (four trials) walking cycle. Four catch trials (that is, without stimulus) served to prevent any anticipation. In addition, the secondary task was performed alone when the subjects were in a seated position. For this static condition, ten stimuli were given following one of five randomly presented preparatory periods: $3,3.5,4,4.5,5 \mathrm{~s}$. The two conditions (one static and one walking) were randomly presented. For the young subjects, there was an additional walking condition in which they had to walk at a speed of about $0.5 \mathrm{~m} / \mathrm{s}$. The same number of trials was used. Subjects were given 10-20 trials of practice with feedback on their speed and then the 25 trials. Trials where speed was not between 4.5 and $5.5 \mathrm{~m} / \mathrm{s}$ were rejected and randomly redone.

\section{Results}

\section{Attentional demands}

The RTs were evaluated in patients and normal subjects when sitting, standing and walking (stimuli were presented at the onset of single-support or double-support phase). The average reaction times (RTs) are illustrated in Figure 1. For the first statistical analysis, only data from sitting, standing and walking at preferred speed were considered. A Group X Task ANOVA with repeated measure shows a significant main effect of Task $(\mathrm{F}(1,42)=38.0$, $P<0.01)$ and a significant interaction Group $\mathrm{X}$ Task
$(\mathrm{F}(1,42)=8.0, \quad P<0.01)$. The decomposition of this interaction into main effects using a Duncan post hoc analysis reveal that normal subjects are slower than the patients when sitting and standing $(P<0.01)$. These results clearly showed that the patients were not affected by their condition in both static tests. However, the patients are significantly slower to answer stimuli than normal subjects when stimuli were presented in the single-support phase $(P<0.01)$ while no significant differences were found in DS although patients were slightly slower than normal subjects $(P>0.05)$. SCI patients performed very well in both static tasks (better than normals) but were significantly slower than normal at preferred speed when walking specially during the single-support phase tasks. When considering the difference in RTs during walking it could be argued that patients have a different walking behavior with one major difference being the speed of progression. In trying to understand the implication of a slower speed in RTs, young subjects were also evaluated during slow, almost equivalent walking speed $(0.5 \mathrm{~m} / \mathrm{s})$. The average RTs are illustrated in Figure 1. For this analysis, only walking conditions were considered and for the normal group, only the slow speed condition was considered. A Group X Task ANOVA with repeated measure shows a significant main effect of Task $(F(1,14)=9.44$, $P<0.01)$ and a significant interaction Group $\mathrm{X}$ Task $(\mathrm{F}(1,14)=14.6, \quad P<0.01)$. The decomposition of this interaction into the main effects using a Duncan post hoc analysis reveal that the patients are faster to answer stimuli when stimuli are presented in DS then normal subjects $(P<0.001)$.

Even though patients compared well with normals walking at slow speed in their RTs, it is possible however to show that relatively, patients need more attention than normals during walking. For the next statistical analysis, a variable was computed with the difference between sitting and walking reaction times to remove individual differences and to illustrate the real attentional cost of walking (Figure 2). For normal subjects, the data from the slow speed are

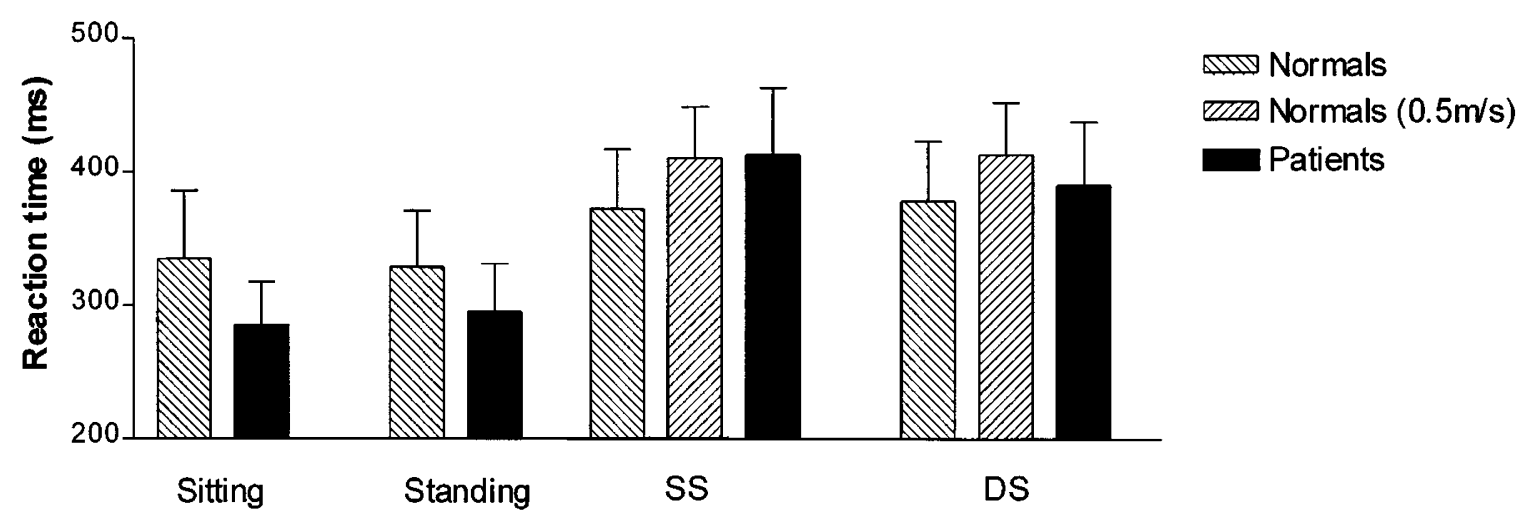

Figure 1 Reaction times for the different experimental conditions: sitting, standing and walking when stimuli were presented in single-support (SS) and double-support (DS), preferred speed and $0.5 \mathrm{~m} / \mathrm{s}$ 
taken because we have already shown a significant difference at preferred speed. An ANOVA was conducted on the transformed walking reaction times. Results showed a significant effect of Group $(\mathrm{F}(1,11)=6.55, \quad P<0.05), \quad$ a significant effect of Support $(\mathrm{F}(1,11)=7.79, P<0.05)$ but no significant interaction. Even if it appears that walking at a slower speed requires more attention in normal subjects than when they walked at their preferred speed, in relative, the patients always allocate more attention to walking than the normal subjects do for both speeds.

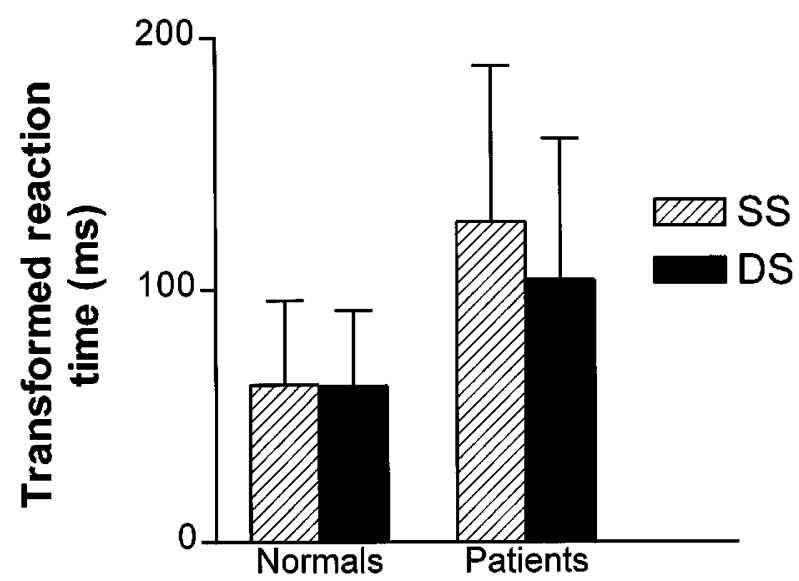

Figure 2 Average transformed reaction times (individual walking reaction times minus individual sitting reaction time) during walking when stimuli were presented in single-support (SS) or double-support (DS). For this picture, only the slower speed $(0.5 \mathrm{~m} / \mathrm{s})$ was used for the normal group

\section{Kinematics of the gait}

Figure 3 presents the average of the cycle length, cycle duration, speed, and percentage of time spent in SS $(\% \mathrm{SS})$ of the patients and the normal subjects when they walked at their preferred speed. The four independent variables were submitted to separate Group X Condition ANOVAs. For all four variables, the main effect of Group reveals significant difference between the two groups for amplitude, duration, speed, and $\% \mathrm{SS}(\mathrm{F}(1,11)=17.49,64.6,170.6,17.8, P<0.01)$. The main effects of Condition, $(\mathrm{F}(1,11)=0.027,0.54$, $1.12, P>0.05$ for length, speed and $\% \mathrm{SS}$ respectively were not significant while duration was $(\mathrm{F}(1,11)=5.88$, $P<0.05)$. The interaction of Group $\mathrm{X}$ Condition, $(\mathrm{F}(1,11)=0.76,0.24,0.38, P>0.05$ for length, speed and $\% \mathrm{SS}$ respectively were not significant while duration was $(\mathrm{F}(1,11)=6.35, P<0.05)$

The kinematic results revealed that the walking behaviors of the young adults were not affected by the stimuli but the patients were (significant interaction for duration). It could be argued that the patients experienced some difficulties performing both tasks (walking and answering the stimuli) together. Normal subjects do not show any difference on the kinematic variable when stimuli are presented or not. Patients tend to increase their speed and decrease their cycle duration when stimuli are presented showing that they might partially lose control of their walking behavior.

In Figure 3, is also presented cycle length, cycle duration, speed, and $\% \mathrm{SS}$ of the normal subjects when they walked at approximately $0.5 \mathrm{~m} / \mathrm{s}$. The four independent variables were again submitted to separate Group X Condition ANOVAs. A significant main effect of Group was found for the duration
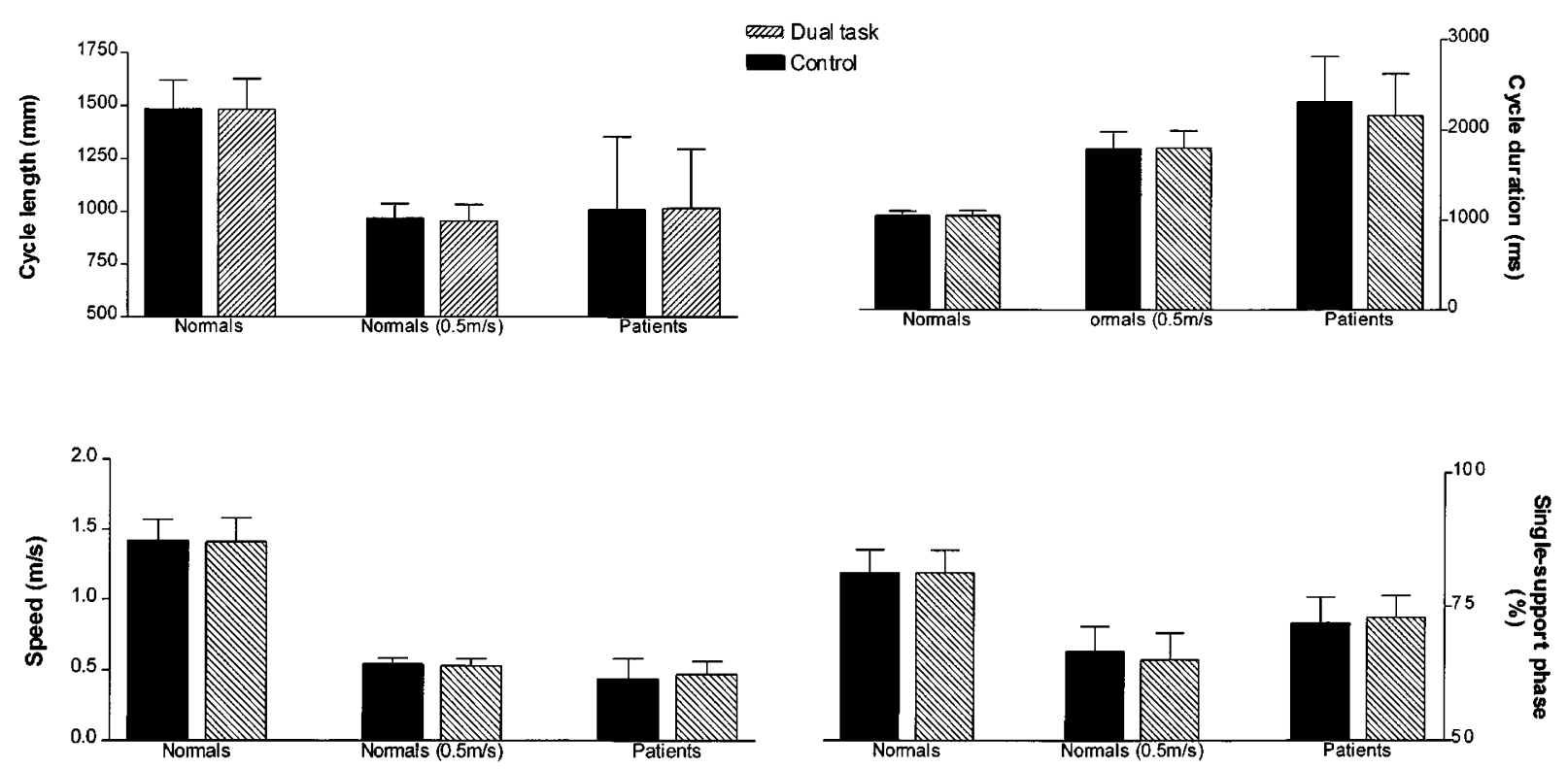

Figure 3 Kinematics of: cycle length, speed, cycle duration and duration of the single-support phase (\% SS) for the two speeds for the normal group and for the SCI subjects 
variable $(\mathrm{F}(1,11)=6.88, P<0.05)$ and for the $\% \mathrm{SS}$ variable $(\mathrm{F}(1,11)=7.91, P<0.01)$. A significant main effect of Condition was also found for the cycle duration variable $(\mathrm{F}(1,11)=5.4, P<0.05)$. The interaction of Group X Condition, was found significant for the duration variable only $(\mathrm{F}(1,11)=6.3, P>0.05)$. The decomposition of the interaction reveals that normal subjects did not modify their cycle duration when stimuli were presented as patients did. Even when walking at a similar speed, some kinematic differences exist between the two groups. The variable $\% \mathrm{SS}$ and the cycle duration illustrate a major difference in the walking pattern between the two groups. Patients seem to spend more time in singlesupport than normals when they walked at a similar speed.

Mean intra-subject variability of two kinematic variables (cycle length and cycle duration) for both groups are presented in Figure 4. Results of standard deviation for the cycle length and cycle duration for the two groups (preferred speed for normals) were subjected to a Group $\mathrm{X}$ Variable ANOVA with repeated measure. Results show a significant main effect of Variable $(F(1,14)=7.11$, $P<0.05)$. No significant effect of group $(\mathrm{F}(1,14)=0.24, P>0.05)$ and no significant interaction $(\mathrm{F}(1,14)=0.62, P>0.05)$ was found suggesting that patients and normal subjects are significantly more variable in cycle duration than in cycle length. Another similar ANOVA was performed, for data when normal subjects were asked to walk at a slower speed (Figure 4). Results show a significant effect of Group $(\mathrm{F}(1,14)=4.04, \quad P<0.05)$ and a significant effect of Variable $(\mathrm{F}(1,14)=33.1$, $P<0.001)$, and no interaction $(\mathrm{F}(1,14)=3.02$, $P>0.05)$. Again, subjects are more variable in cycle duration than in cycle length. Furthermore, normal subjects when they walked at a slower speed are more variable than patients are.

\section{Discussion}

Results of RTs reveal that patients need to allocate significantly more of their attentional resources to walking than normal subjects. Several arguments may explain this difference. SCI patients have less control on their action mainly because of postural instability, lack of equilibrium, muscle weakness, sensory lost and spasticity. To counterbalance those problems, they must closely monitor their movements and evaluate the impact of those in the environment. This evaluation requires more attentional resources given to sensory integration (visual, vestibular and proprioceptive). Unfortunately, the protocol used in this experiment could not determine if patients have reached the limit of their attentional resources when walking but nevertheless, a substantial amount of attention (more than the young adults tested) is required. When compared to normal subjects at the same walking speed (approximately $0.5 \mathrm{~m} / \mathrm{s}$ ), patients compare well in terms of reaction times. However, when we look at the relative cognitive involvement, it can be shown that, again, patients need more attentional resources for walking. For both speeds, normals show no difference whether the stimulus is presented in SS or in DS. However, patients showed a significant difference between the two support phases. Patients are more affected than normals when stimuli are presented during the single-support phase when compared to when presented in doublesupport phase. This result indicates that the singlesupport phase is more attention demanding than the double-support phase. Kinematics variables may partially explain this difference. When walking at preferred speed, young adults showed several differences: longer cycle length, higher speed, shorter cycle duration and higher cadence. Even when walking at similar speed, young adults produce shorter cycle duration and spend less time in single-support phase
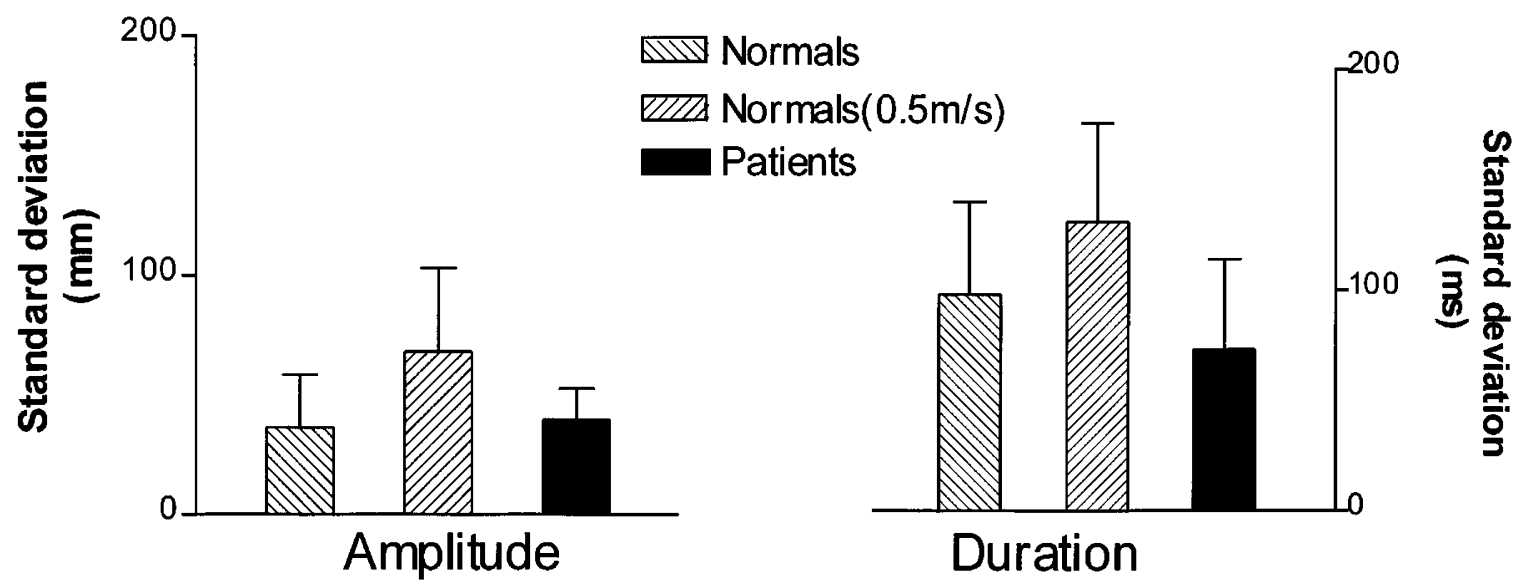

Figure 4 Variability of the two groups at preferred speed and for the normal group at $0.5 \mathrm{~m} / \mathrm{s}$, for the cycle amplitude and cycle duration 
in comparison with the patients illustrating that the difference between the two groups is not only speed, but also walking behavior. This could illustrate that the SS phase for patients is more difficult to produce and certainly not completely ballistic as suggested by Cappozzo et al; ${ }^{12}$ Mochon and McMahon. ${ }^{13}$ For patients, SS could also be interpreted as a pseudo DS because of the use of a walking device. They are rarely in SS if the number of ground contact is taken into account. Several patients take more time to initiate their SS having sometime to lean forward to permit the toes to swing forward.

Patients also showed some deficit in the realization of a fluid walking behavior when compared to normals. Furthermore, even when initiated, the swing phase in the patients is often characterized by some unwanted contacts with the ground which add to the encountered difficulties. Patients also presented some equilibrium difficulties in the DS phase and do not want to spend a long period of time in this position. SCI subjects when walking will produce several discreet walking cycles, not as continuous as a normal adult would do. Between cycles, the reorganization of the walking devices is made and equilibrium is reached before the initiation of the subsequent cycle. Patient's walking is saccadic rather than continuously performed.

Finally, when variability is considered both groups when walking at preferred speed showed similar intrasubject variability. Both groups have been walking at their preferred speed and seem reasonably constant in their 'normal' walking behavior, and even patients seem to adopt a relatively constant walking behavior according to their condition (Figure 3). Difference in RTs can not be attributed only to difficulty to produce walking behavior (very low variability) but also to equilibrium demands resulting mostly from slower speed. Normal subjects are significantly more variable than patient when they walked at a slower speed showing that walking at a speed different from the preferred one leads to an important increase of variability. Furthermore, it can be argued that slower speed increase equilibrium demands which can be seen in $\mathrm{RTs}$ results.

SCI subjects have some walking capability. However, this capability is greatly affected by weakness, spasticity, and lack of postural control and equilibrium reducing the automaticity of the walking behavior. Walking for SCI subjects requires more cognitive involvement than for normal subjects. The complexity of the walking behavior for SCI patients using walking aids might be another factor related to slower reaction times (further study on how walking aids affect RTs should be made). However, increase in attentional demands could not be attributed to the use of walking aids only because those patients without the use of such aids would have precarious equilibrium that would require more attentional resources. Measuring the cognitive involvement during walking in SCI patients could lead to measure to what extent walking is automatic and even what type of walking aids a patient should adopt.

\section{Acknowledgements}

The project was supported by grants from the Network of Centres of Excellence (NCE) on neural regeneration and functional recovery to Dr Yves Lajoie.

\section{References}

1 Barbeau H, Rossignol S. Enhancement of locomotor recovery following spinal cord injury. Curr Opin Neurol 1994; 7: 517-524.

2 Barbeau $\mathrm{H}$ et al. Walking following spinal cord injury: Control and recovery. The Neuroscientist 1998; 1: 1-11.

3 Burke DC, Burley HT, Ungar GH. Data on spinal injuries: Part II. Outcome of the treatment of 352 consecutive admissions. Aust $N Z J$ Surg 1985; 55: $377-382$.

4 Rossignol $\mathrm{S}$ et al. Locomotor capacities after complete and partial lesion of the spinal cord. Acta Neurobiological Exp 1996; 56: $449-463$

5 Forssberg H. Brain stem control of spinal mechanisms. In: Sjolund B, Bjorklund A (eds). Spinal locomotor functions and descending control. Elsevier: Biomedical Press, 1982; pp. $253-$ 271.

6 Grillner S, Wallen P. Central pattern generators for locomotion, with special reference to vertebrates. Annu Rev Neurosci 1985; 8: $233-261$

7 Lajoie Y, Teasdale N, Bard C, Fleury M. Attentional demands for static and dynamic equilibrium. Exp Brain Res 1993; 97: 139 144.

8 Lajoie $\mathrm{Y}$ et al. Gait of a deafferented subject without large myelinated sensory fibers below the neck. Neurology 1996; 47: $109-115$.

9 Barbeau H, Fung J. Recovery of locomotion following spinal cord injury: new concepts and approaches in rehabilitation. In: Couch JR, Good DC (eds). Handbook of Neurorehabilitation. Dekker Inc: New York 1994; pp. 73-104.

10 Kahneman D. Attention and effort. Englewood Cliffs, NJ: Prentice-Hall, 1973.

11 Abernethy B. Dual-task methodology and motor skills research: some applications and methodological constraints. J Hum Mov Stud 1988; 14: $101-132$.

12 Cappozzo A, Figura F, Marchetti M. The interplay of muscular and external forces in human ambulation. $J$ Biomech 1976; 9: 35 43.

13 Mochon S, McMahon TA. Ballistic walking. J Biomech 1980; 13: $49-57$. 